\title{
Elastic and plastic-flow damage constitutive model of rock based on conventional triaxial compression test
}

\author{
Pingyuan Yang ${ }^{1}$, Xiaoen $\mathrm{Wu}^{2 *}$, Junhua $\mathrm{Chen}^{3}$ \\ ${ }^{1}$ Institute of Mineral Resources, Chinese Academy of Geological Sciences, Beijing 100037, China \\ ${ }^{2}$ China Energy Engineering Group Equipment Co. Ltd., Beijing 100044, China \\ ${ }^{3}$ School of Civil Engineering, Central South University, Changsha 410075, China
}

Corresponding Author Email: xewu@ceec.net.cn

https://doi.org/10.18280/ijht.360320

Received: 17 January 2018

Accepted: 25 May 2018

\section{Keywords:}

rock, damage mechanics, strain softening, brittleness, plastic flow, dilatancy

\begin{abstract}
This study summarizes the stress-strain relations of rocks in the conditions of conventional triaxial compression test. Combining the two mechanisms of damage and plasticity, this study explores inner mechanism of stress-strain relation and analyzes the applicability and limitation of the classical constitutive models of linear elasticity-plasticity, nonlinear elasticity and elastic damage. Based on considering the advantages and disadvantages of classical constitutive models, classical plastic statistic damage models are improved. In addition, this study puts forward the constitutive model of plastic statistic damage simultaneously considering plastic flow and damage function, deduces constitutive relational expressions in the conditions of conventional triaxial compression test and preliminarily verifies the constitutive relational expressions using the result of conventional triaxial compression test. In research result, the constitutive model improved can well and comprehensively reflect strain softening and dilatancy of rock and reflect rocks' character of transforming from brittle to plastic flow following the increase of confining pressure.
\end{abstract}

\section{INTRODUCTION}

Rock is the most common material in civil engineering and construction. Its constitutive theory is a research hotspot. At present, the classical isotropic elastic-plastic mechanics theory is still the theoretical basis for studying the constitutive relation of rock. According to this theory, rock is mainly elastic before the internal structure is destroyed. After the damage occurs, its mechanical properties are between solid and fluid. The deformation characteristics are mainly plastic flow. In large quantity of tests and researches [1-4], the classical elastic-plastic constitutive theory that is suitable for metal materials is not completely suitable for rock. For example, rock's character of hardness, yielding character in stagnant water pressure conditions, dilatancy character and strain softening character in trans-pression state are not those of common metal materials. The essence of mechanical property differences between rock and metal is that rock is a heterogeneous continuum containing gaps and crack. Therefore, it is difficult to build an isotropic constitutive model that can completely reflect above mechanic characters of rock and can be used conveniently. Usually, the more influencing factors considered, the more parameters there will be and more complex constitutive model will be. A lot of researches show [5-9] that the common method of building constitutive model of rock is to introduce necessary theory or hypothesis as supplement on the basis of classical elasticplastic mechanics theory. In this way, constitutive model can describe main characters of rock as many as possible and accurately forecast strain of rock and stress-strain relation based on the premise of small quantity of calculation parameters.
Damage mechanics theory is widely used in rock engineering. Many scholars combine damage mechanics theory and basic theory of elastoplasticity to build constitutive model of elastic damage or elastic-plastic damage [10-11]. Considering that rock deformation and damage in engineering application scope is reflected by strain softening and brittle damage, many scholars use constitutive theory of elastic damage to build constitutive model of rock [12]. It is a common constitutive theory of elastic damage to introduce constitutive theory of elastic statistic damage formed by microscopic statistic damage theory into constitutive theory of classical elastic damage. Constitutive theory of elastic statistic damage has got good effect in the aspect of forecasting axial strain softening of rock. In addition, comparing with other constitutive models of elastic damage, the number of calculation parameters of the constitutive model is smaller and mathematical expression of the model is simpler. Therefore, constitutive model of elastic statistic damage can be conveniently used in engineering. However, generally, the Poisson's ratio in the constitutive model of elastic statistic damage does not change with the damage. In this case, rock dilatancy property in trans-pression state cannot be considered for the constitutive model. For example, isotropous elastic damage statistic constitutive model built by Cao et al[17]can well simulate axial strain softening in trans-pression state but cannot reflect the property of volume dilatancy. In addition, like other solids, as the confining pressure increases, the rock will gradually exhibit some mechanical properties similar to those of fluids, such as plastic flow. This is also ignored by the classical elastic damage model. The researches show [13-15] that most damage constitutive models cannot simultaneously 
reflect strain softening, volume dilatancy, and plastic flow of rock.

In practical engineering, rock is usually pressed in several directions. Therefore, triaxial compression test is usually used to research the constitutive relation of rock. In large quantity of researches, true triaxial test can better explain the law of rock deformation and damage in pressure than conventional triaxial test, but the equipment of true triaxial test is expensive and conventional triaxial test is usually operated to get mechanical parameters of rock in practical engineering. At present, conventional triaxial test is usually operated to research the constitutive relation of rocks.

Aiming at the defect of existing researches and for the convenience of engineering application, this study tries to build a constitutive model of elastic-plastic damage that can sufficiently and correctly reflect rock dilatancy, strain softening and plastic flow based on classical elastic statistic damage theory. The computed result got from constitutive model is compared with the result of conventional triaxial test. In the comparison and analysis, rationality of the model is verified to offer reference to the research on constitutive model of rock.

\section{ANALYSIS AND DISCUSSION ON ROCK STRAIN AND STRESS-STRAIN RELATION BASED ON DAMAGE AND PLASTIC FLOW}

\subsection{Summary of rocks' strain and stress-strain relation}

In large quantity of tests and researches, although deformation and damage of rocks depend on internal structural features and mineral components of rocks, rocks in different variety have different strain and stress-strain relation. The deformation and damage of rocks in the nature still have their basic similarities. In conventional triaxial compression, axial stress and axial stress-strain relation curve of rocks have two forms of strain softening and strain hardening. Strain hardening curve corresponds to the rock deformation under high confining pressure, including compaction stage, linear elasticity stage and non-elastic hardening stage. Strain softening curve corresponds to the rock deformation in low confining pressure. The curve before peak value of strain is divided into compaction stage, linear elasticity stage and nonelastic hardening stage; there is a strain softening stage after peak value of strain. For the convenience of analysis, the strain and stress-strain relation curve in the two types are simplified in Figure 1. Curve OACD is for the type of strain hardening and curve OABEF is for the type of strain softening in Figure 1. Curve $\mathrm{OA}_{1} \mathrm{C}_{1} \mathrm{D}_{1}$ is the volume strain and axial strain relation curve of the rocks of strain hardening type. Curve $\mathrm{OA}_{1} \mathrm{~B}_{1} \mathrm{~F}_{1}$ is the volume strain and axial strain relation curve of the rocks of strain softening type. There are the following laws:

(1) Under low confining pressure, strain and stress-strain relation curve is in the type of strain softening. As for curve $\mathrm{OABEF}$, point $\mathrm{C}$ is the peak point that represents damage strength; EF segment corresponds to strain softening stage in which strain and stress-strain relation develops unstably and rocks are in brittle damage. Under high confining pressure, strain curve of rocks is in the type of strain hardening. As for the strain curve OACD of rocks, rocks are in dilatant damage. With the increase of confining pressure, damage strength of rocks and strain of corresponding damage point increase. Rock deformation transforms from strain softening to strain hardening. At the same time, the mode of rock damage is transformed from brittleness to plastic flow.

(2) OA is the closure stage of the internal defects (cavity and cracks) of rocks. It is common in the deformation process of the rocks with high voidage. As for the rocks with low voidage and compact hard rocks, the stage can be ignored. Linear elastic stage is after the closure stage. In Figure 1, they respectively correspond to $\mathrm{AB}$ segment under low confining pressure and $\mathrm{AC}$ segment under high confining pressure. Stress-strain relation in the stage follows Hooke's law. The strain level at elastic limiting point rises following the increase of confining pressure, such as the comparison between point $\mathrm{C}$ and point $\mathrm{B}$. Starting from elastic limiting point, the stressstrain relation of rocks becomes complex. Stress-strain relation shows its nonlinear change trend; dilatancy of volume begins, such as $\mathrm{C}_{1} \mathrm{D}_{1}$ segment in Figure 1.

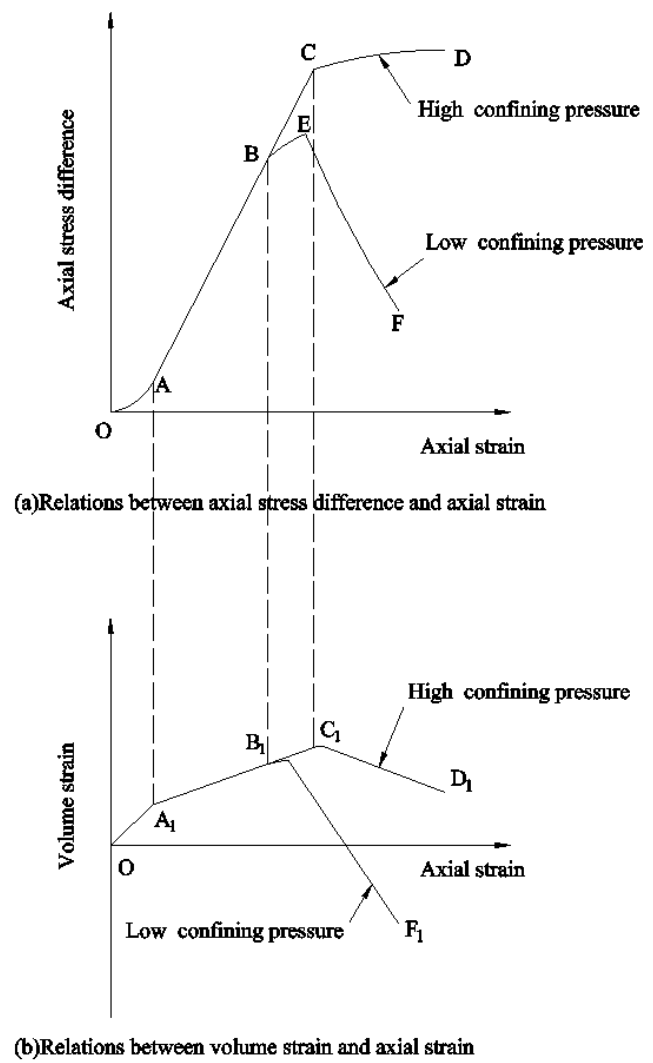

Figure 1. Stress-strain relations of rock under conventional triaxial compression test

(3) Generally speaking, volume of rocks is firstly compressed and then dilatated. Linear elastic limiting point is the starting point of dilatancy. For example, point $\mathrm{B}$ and $\mathrm{B}_{1}$ are the starting points of dilatancy in Figure 1. In other words, after loading strain exceeds linear elastic limiting point, axial stress and nonlinear deformation follow dilatancy of rocks, such as stress-strain relation curves BEF and BCD and corresponding volume strain and axial stress-strain relation curves $\mathrm{B}_{1} \mathrm{~F}_{1}$ and $\mathrm{C}_{1} \mathrm{D}_{1}$. They are in the stage of dilatancy. Generally speaking, strain hardening process accompanies small capacity of volume dilatancy; strain softening accompanies large capacity of volume dilatancy, such as the comparison between curve $\mathrm{B}_{1} \mathrm{~F}_{1}$ and curve $\mathrm{C}_{1} \mathrm{D}_{1}$. This is caused by the inhibiting effect of confining pressure to dilatancy of rocks. 


\subsection{Analysis on mechanical mechanism of rocks' stress- strain relation}

(1) Damage mechanical mechanism

In the aspect of internal structure, as for complete damagefree rocks, their mineral components and crystalline interface of mineral grains are decisive factors which influence mechanical property of rocks. Under normal conditions, crystalline interface is the weak interface in damage-free rocks. As for initially-damaged rocks, defects of cavity and cracks are also the weak parts of rocks. These parts of rocks can be easily damaged. The local damages gradually evolve and eventually influence stability of macroscopic entirety. According to the basic theory of damage mechanics, internal damages of rocks develop at the weak parts. Extension of damage is assumed to be microcrack. In other words, damage extends at the weak parts in the form of microcrack. As for the strain softening curve OABEF in Figure 1, in the function of external loading, development of microcracks in rocks is respectively in compact dilatancy-free stage, stable dilatancyfree stage, stable dilatancy stage and non-stable dilatancy stage corresponding to $\mathrm{OA}$ segment, $\mathrm{AB}$ segment, $\mathrm{BE}$ segment and $\mathrm{CF}$ segment. Non-stable dilatancy stage of microcrack accompanies the cracks in macroscopic form. At this time, rocks are in non-stable damage stage. As for strain hardening curve, there is no unstable stage of stress-strain relation because of the restraint of high confining pressure. In other words, with increase of confining pressure, the probability of macroscopic crack lowers.

The change of microcrack (including compaction and dilatancy) is always followed by irreversible deformation. Therefore, except for AB elastic stage of stable dilatancy-free stage of microcrack, there is irreversible volume deformation in other stages. In conventional triaxial compression conditions, microcrack dilates in axial compression direction; deformation in side direction is faster than that in axial direction. So, in damage process, dilatancy in side direction is large and dilatancy of rock is caused, such as the curve $\mathrm{B}_{1} \mathrm{D}_{1}$ in Figure 1. Confining pressure can restrain or constrain the dilatancy of microcrack. Therefore, when confining pressure is large, dilatancy can be restrained, such as the comparison between curve $B_{1} F_{1}$ and curve $C_{1} D_{1}$ in Figure 1 . With the increase of stress, microcracks dilate. Dilatancy of microcrack will cause release of stress and nominal stress decreases. Stress-strain relation become non-linear.

Therefore, damage and the volume dilatancy caused by damage increase following the increase of loading stress and decrease following the increase of confining pressure.

(2) Mechanism of plastic flow. Existing microscopic test shows [16] that plastic deformation area around internal microcrack area of solid can be always observed. If unstable dilatancy of microcracks causes the formation of macroscopic cracks and any plastic flow is not observed, the damage is called brittle damage. In fact, solid materials have no absolute brittle damage. Therefore, in rock damage process, there is more or less plastic flow, showing some fluid properties. The more complete the rock is or the smaller the initial damage is, the easier it is to exhibit plastic flow. The irreversible deformation of rocks can be divided to be damage and plastic flow. Under low confining pressure, the irreversible deformation of rocks is mainly caused by damage. The way the rock breaks down is brittle failure. Under high confining pressure, damage development is restrained. Irreversible deformation is caused by plastic flow. Rock shows its flow deformation that is similar to that of fluids.

In general, the nonlinear deformation and damage of rocks are determined by two mechanisms of damage and plastic flow. They are competitive to each other. When damage dominates prominently, both the strain softening and dilatancy degree of rocks are conspicuous. Conversely, if plastic flow dominates, strain softening transforms to strain hardening and dilatancy becomes weak. Their domination in damage mechanism depends on the level of confining pressure.

\section{APPLICABILITY AND LIMITATION OF EXISTING CONSTITUTIVE MODELS OF ROCKS}

\subsection{Classical elastic-plastic constitutive model of rocks}

Linear elastic-plastic constitutive theory, nonlinear elastic constitutive theory and constitutive theory of elastic damage are those applied widely at present. General mathematical expressions of these constitutive theories are given below:

(1) General mathematical expressions of linear elasticplastic constitutive model:

$$
\begin{gathered}
\mathrm{d} \sigma_{i j}=D_{i j k l}^{\mathrm{e}} \mathrm{d} \varepsilon_{k l}^{\mathrm{e}} \\
\mathrm{d} \varepsilon_{i j}=\mathrm{d} \varepsilon_{i j}^{\mathrm{e}}+\mathrm{d} \varepsilon_{i j}^{\mathrm{p}} \\
\mathrm{d} \varepsilon_{i j}^{\mathrm{p}}=\lambda \frac{\partial f_{\mathrm{p}}\left(\sigma_{i j}\right)}{\partial \sigma_{i j}} \\
f_{\mathrm{p}}\left(\sigma_{i j}\right)-H=0
\end{gathered}
$$

Where, $\sigma_{i j}$ is nominal stress tensor; $\varepsilon_{i j}$ is strain tensor; $\varepsilon_{i j}^{\mathrm{e}}$ is elastic strain tensor; $\varepsilon_{i j}^{\mathrm{p}}$ is plastic strain tensor; $D_{i j k l}^{\mathrm{e}}$ is rigidity tensor of linear elastomer. $f_{1}\left(\sigma_{i j}\right)$ is the yielding function about stress tensor $\sigma_{i j}$ in stress space; $\lambda$ is plastic factor; $H$ is hardening parameter.

(2) General mathematical expressions of nonlinear elastic constitutive model:

$$
\begin{aligned}
& \mathrm{d} \sigma_{i j}=D_{i j k l}^{\mathrm{n}} \mathrm{d} \varepsilon_{k l} \\
& D_{i j k l}^{\mathrm{n}}=f_{\mathrm{n}}\left(\varepsilon_{k l}\right)
\end{aligned}
$$

where, $D_{i j k l}^{\mathrm{n}}$ is elastic tangential rigidity tensor; $f_{\mathrm{n}}\left(\varepsilon_{k l}\right)$ is the function with independent variable of strain tensor in strain space.

(3) Constitutive model of elastic damage:

$$
\begin{aligned}
& \mathrm{d} \sigma_{i j}^{\prime}=D_{i j k l}^{\mathrm{e}}(1-\omega) \mathrm{d} \varepsilon_{k l} \\
& \omega=f_{\mathrm{d}}\left(\sigma_{i j}^{\prime}\right) \\
& \sigma_{i j}^{\prime}=\sigma_{i j}(1-\omega)
\end{aligned}
$$

where, $\omega$ is damage variable or damage factor; $\sigma_{i j}^{\prime}$ is effective 
stress tensor; $f_{d}\left(\sigma_{i j}^{\prime}\right)$ is the function with the dependent variable of damage variable. It is damage evolution expression, generally.

For strict deduction of constitutive theory, mature mechanics theory basis is needed for building ideal elasticplastic constitutive model and constitutive model of elastic damage, including certain reasonable hypothesis. For example, plastic flow and damage function mechanism are respectively considered on the basis of linear elastic law. Nonlinear elastic constitutive model starts from testing phenomenon and mainly aims at the nonlinear phenomenon of rock deformation mechanics. Its mathematical meaning is more than physical meaning. Generally, it is a classical model by test data fitting.

From the angle of building mathematical expression of constitutive model, nonlinear elastic constitutive model is simplest. Generally, the mathematical expression that meets stress-strain relation in test is needed only. Elastic damage model is slightly complex and elastic-plastic model is the most complex one. However, as for nonlinear elastic model, the more complex testing phenomenon is, the more influencing factors of building constitutive model shall be considered. Calculating parameters in constitutive model are more than those of other two constitutive models, correspondingly. From the angle of application scope, the nonlinear elastic model based on test summary is only applicable to the rocks with macroscopic same deformation and damage mechanism in the test stress path. Elastic-plastic model and elastic damage model deduce common stress-strain relation of mechanical unit from microscopic mechanics. They are applicable to describing the deformation and damage of rocks in different varieties.

Nonlinear elastic model is simple. Many nonlinear elastic constitutive models can be even written to be in population parameter form and explicit solution can be got easily. As for elastic-plastic model and elastic damage model, especially in complex stress conditions, iteration calculation may be needed in calculation process. So, the process of calculating elasticplastic model and elastic damage model is complex.

\subsection{Stress-strain relation of rocks in classical constitutive theory}

The ideal elastic-plastic constitutive model (hereinafter called MC constitutive model) with the yielding condition of Mohr-Coulomb damage rule and Duncan-Chang hyperbola model (hereinafter called DC constitutive model) are common constitutive models among elastic-plastic constitutive models and nonlinear elastic constitutive models, respectively. To conveniently analyze and discuss advantages and disadvantages of existing constitutive models, MC constitutive model and DC constitutive model are used in this study to represent elastic-plastic constitutive model and nonlinear elastic constitutive model, respectively. The constitutive model of elastic statistic damage built by Cao Wengui is chosen as constitutive model of elastic damage. For details, please refer to Cao and $\mathrm{Li}$ [17].

In conventional triaxial conditions, the constitutive relational expression got from different models are calculated below:

(1) MC constitutive model:

Yielding condition:

$$
f_{\mathrm{p}}=\sigma_{1}(1-\sin \varphi)-\sigma_{3}(1+\sin \varphi)-2 c \cos \varphi=0
$$

Axial stress and axial strain:

$\mathrm{d} \varepsilon_{1}=\frac{\mathrm{d}\left(\sigma_{1}-\sigma_{3}\right)}{E}+\mathrm{d} \varepsilon_{1}^{\mathrm{p}}=\frac{\mathrm{d} \sigma_{1}}{E}+\lambda \frac{\partial f_{\mathrm{p}}}{\partial \sigma_{1}}$

plastic volume strain:

$\mathrm{d} \varepsilon_{\mathrm{v}}^{\mathrm{p}}=\mathrm{d} \varepsilon_{1}^{\mathrm{p}}+2 \mathrm{~d} \varepsilon_{3}^{\mathrm{p}}=\lambda \frac{\partial f_{\mathrm{p}}}{\partial \sigma_{1}}+2 \lambda \frac{\partial f_{\mathrm{p}}}{\partial \sigma_{3}}$

(2) DC constitutive model:

Axial stress and axial strain:

$\sigma_{1}-\sigma_{3}=\frac{\varepsilon_{1}}{a+b \varepsilon_{1}}$

$E=\frac{1}{a}=k_{1} p_{0}\left(\sigma_{3} / p_{0}\right)^{k_{2}}$

$\frac{1}{b}=\frac{2 c \cos \varphi+2 \sigma_{3} \sin \varphi}{(1-\sin \varphi) k_{3}}$

Axial strain and volume strain:

$\varepsilon_{1}=\frac{-\left(\varepsilon_{\mathrm{v}}-2 \varepsilon_{1}\right)}{k_{4}+k_{5}\left(\varepsilon_{\mathrm{v}}-2 \varepsilon_{1}\right)}$

$k_{4}=k_{6}+k_{7} \lg \sigma_{3}$

(3) Constitutive model of elastic damage:

Axial stress and axial strain:

$\mathrm{d}\left(\sigma_{1}-\sigma_{3}\right)=E(1-\omega) \cdot \mathrm{d} \varepsilon_{1}-\mathrm{d} \omega \cdot E \varepsilon_{1}$

$\omega=1-\exp \left[-m_{1}\left(f_{\mathrm{p}} / 1-\omega\right)^{m_{2}}\right]$

Axial strain and volume strain:

$\mathrm{d} \varepsilon_{\mathrm{v}}=(1-2 \mu) \mathrm{d} \varepsilon_{1}$

In mathematical expressions (10)-(20): $\sigma_{1}>\sigma_{2}=\sigma_{3}, \sigma_{1}$ is the largest main stress; $\sigma_{2}$ is middle main strain; $\sigma_{3}$ is the smallest main stress. $\varepsilon_{1}>\varepsilon_{2}=\varepsilon_{3}, \varepsilon_{1}$ is the largest main strain; $\varepsilon_{2}$ is middle main strain; $\varepsilon_{3}$ is the smallest main strain; $\varepsilon_{\mathrm{v}}$ is volume strain; $\varepsilon_{v}^{p}$ is plastic volume strain; $\varepsilon_{1}^{p}$ is axial plastic strain(the largest main plastic strain); $\varepsilon_{3}^{p}$ is lateral plastic strain(the smallest main plastic strain); $\varphi$ is internal friction angle; $c$ is cohesion force; $E$ is nondestructive elastic modulus; $\mu$ is nondestructive Poisson's ratio; $k_{1}, k_{2}, k_{4}$ and $k_{5}$ are fitting parameters; $k_{3}$ is strength reduction factor. $0.75 \leq k_{4} \leq 0.95 ; p_{0}$ is reference pressure or atmospheric pressure. Its approximate value can be $p_{0}=100 \mathrm{kPa} . m_{1}$ and $m_{2}$ are probability distribution parameters.

From above relational expressions, it is known that the number of parameters of MC model is smallest, including 4 parameters in total: 2 elastic parameters of $E$ and $\mu$ and 2 yielding parameters of $\varphi$ and $c$. Besides above 4 parameters, 
elastic damage model also contains two probability distribution parameters: $m_{1}$ and $m_{2}$. There are 6 parameters in total. The number of parameters for DC model is the largest. There are 8 fitting parameters in total. After parameters are given, constitutive models can be used to forecast stress-strain relation.

Figure 2 is conventional triaxial compression stress-strain relation curve got from constitutive models including MC model based on the parameters of elasticity modulus, Poisson's ratio, damage strength and damage strain.

In comparison with Figure 1, it is known that $\mathrm{MC}$ model ignores nonlinearity of rocks' stress-strain relation before and after peak value of stress based on the assumption that rock is linear elastic entity before yielding and strain hardening or strain softening is not considered in yielding stage. However, plastic volume dilatancy is considered in yielding stage of MC model. Therefore, rock dilatancy can be forecast. Since the MC model uses the associated flow rule, the deformation of the predicted dilatancy is too large. Although nonlinear variation trend is considered in DC model, it ignores straight segment of linear development in rocks' stress-strain relation and exaggerates nonlinear deformation of rocks. So it is not applicable to hard rocks. In addition, DC model cannot forecast dilatancy of rocks. Aiming at axial deformation forecast, rocks' characters in linear elastic stage and nonlinear stage are sufficiently considered in elastic damage model. It is suitable for forecasting hardening and strain softening of soft rocks or hard rocks before and after stress value. Being same as DC model, volume deformation only has compression stage and the dilatancy of rocks cannot be effectively forecast. Besides, the three models cannot forecast compaction of rocks.

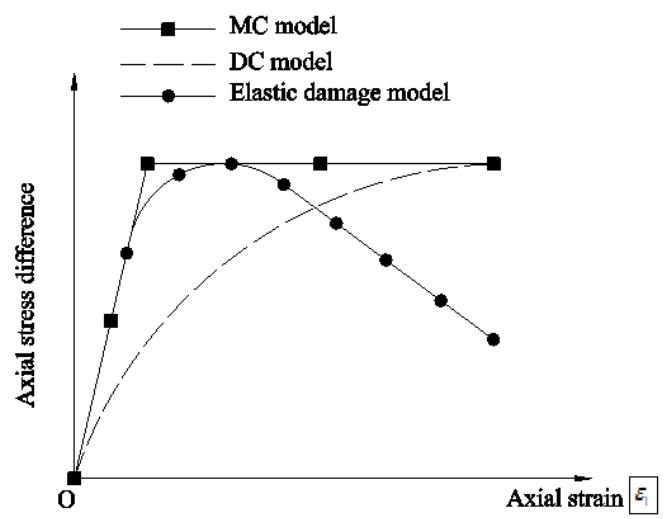

(a)Relations between axial stress difference $\left(\sigma_{1}-\sigma_{3}\right)$ and axial strain $\varepsilon_{1}$

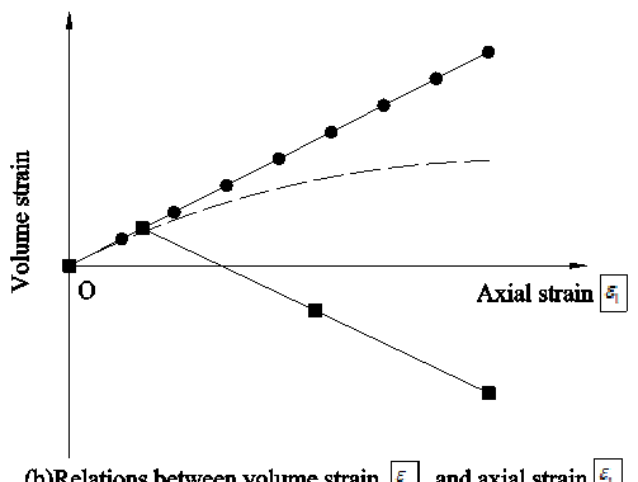

Figure 2. The forecast of stress-strain relation based on classical constitutive theory
Based on above analysis on the mechanism of influencing rocks' stress-strain relation, plastic irreversible deformation after stress peak is considered in MC model, such as plastic volume deformation of dilatancy. However, damage mechanism is not considered. So nonlinear relation in overall stress-strain relations of rocks is not reflected in MC model. DC model is suitable for forecasting nonlinear segment of stress-strain relation under high confining pressure, such as CD segment in Figure 1. Although the mechanism of damage causing release of stress is considered in elastic damage model that well simulates rocks' stress-strain nonlinear variation before and after stress peak, such as the curve BEF in Figure 1 , but it ignores the mechanism of plastic flow and the mechanism of damage accompanying irreversible deformation. So volume dilatancy and mechanical behavior in compaction stage cannot be forecast.

\section{IMPROVEMENT OF CONSTITUTIVE MODEL}

Based on above analyses, to comprehensively reflect rocks' stress-strain relation, irreversible deformation caused by damage function and plastic flow shall be considered simultaneously while building constitutive models. Considering large quantity of calculating parameters of nonlinear elastic model and ambiguous physical significance, to effectively use existing constitutive theories, constitutive models can be built considering the advantages and disadvantages of elastic damage model and MC model. This study improves elastic damage model based on introducing plastic flow mechanism. Yielding condition is the expression (10) in MC model. Except for some rocks with high voidage, the compaction deformation of most rocks is small. The constitutive model improved ignores the compaction segment $\mathrm{OA}$ in Figure 1.

\subsection{Assumption of deformation coupling mechanism of plastic flow and damage function}

In strict significance, damage causes inconsecutive irreversible deformation of rocks. Existing plastic flow theory is put forward aiming at irreversible deformation of continuous solid medium. Therefore, the two deformations exist simultaneously but their mechanisms are different. In the assumption of isotropic continuum, being similar to relation between viscous deformation and plastic deformation in viscous-plastic theory, it can be assumed that damage process accompanies plastic flow; irreversible deformation of damage is plastic irreversible deformation. Based on above assumption, the following relational expression is established to describe deformation:

$\mathrm{d} \varepsilon_{i j}=\mathrm{d} \varepsilon_{i j}^{\mathrm{e}}+\mathrm{d} \varepsilon_{i j}^{\mathrm{p}}=\mathrm{d} \varepsilon_{i j}^{\mathrm{e}}+\mathrm{d} \varepsilon_{i j}^{\omega}$

where, $\varepsilon_{i j}^{\omega}=\varepsilon_{i j}^{\mathrm{p}}, \varepsilon_{i j}^{\omega}$ is irreversible strain tensor caused by damage.

\subsection{Compatibility conditions in irreversible deformation stage}

(1) Damage evolution condition

According to damage statistics theory, it is assumed that probability of damage of infinitesimal single rock following 
plastic flow satisfies Weibull distribution. The formulae of rock damage evolution:

$$
\begin{aligned}
& \omega=p_{\mathrm{f}}=1-\exp \left[-\left(\bar{\gamma}^{\mathrm{p}} / a\right)^{b}\right] \\
& \bar{\gamma}^{\mathrm{p}}=\int\left[(2 / 3) \cdot \mathrm{d} e_{i j}^{\mathrm{p}} \cdot \mathrm{d} e_{i j}^{\mathrm{p}}\right]^{\frac{1}{2}}
\end{aligned}
$$

where, $p_{\mathrm{f}}$ is damage probability; $\bar{\gamma}^{p}$ is equivalent shearing plastic deformation; $e_{i j}^{\mathrm{p}}$ is plastic deviatoric strain tensor; $a$ and $b$ are parameters of Weibull distribution. $a>0, b<0$.

According to, both $a$ and $b$ are relevant to confining pressure [18]. The relational expressions between them are:

$$
\begin{aligned}
& a=n_{1}\left(\sigma_{3} / p_{0}\right)+n_{2} \\
& b=-n_{3}\left(\sigma_{3} / p_{0}\right)+n_{4}
\end{aligned}
$$

where, $n_{1}, n_{2}, n_{3}$, and $n_{4}$ are fitting parameters and all of them are larger than 0 .

From (22)-(25), it is known that: the larger plastic deformation $\bar{\gamma}^{p}$ is, the larger probability of damage of infinitesimal rock will be. When damage is accumulated to be destruction, if plastic deformation $\bar{\gamma}^{p}$ is small, the mode of rock destruction is brittle destruction. Conversely, the mode of rock destruction is dilatant plastic flow. Besides, the larger confining pressure $\sigma_{3}$ is, the more difficult increase of damage value $\omega$ will be. When damage is accumulated to be destruction, plastic deformation increases correspondingly. These damage evolution characters meet the laws of rock deformation and damage summarized above.

(2) Plastic flow conditions

Based on above analyses, the yielding condition determined by formula (10) is ideal plasticity. It is not proper to reflect yielding hardening. Also considering correction of damage to stress in formula (9), the yielding function of formula (10) is modified and deduced to get the following yielding function expression:

$$
\begin{aligned}
& f_{\mathrm{p}}\left(\sigma_{i j}^{\prime}, H\right)=f_{\mathrm{p}}\left(\sigma_{i j}, \omega, H\right)=0 \\
& =\sigma_{1}^{\prime}(1-\sin \varphi)-\sigma_{3}^{\prime}(1+\sin \varphi)-H \\
& =\frac{\sigma_{1}(1-\sin \varphi)-\sigma_{3}(1+\sin \varphi)}{1-\omega}-H
\end{aligned}
$$

$$
H=2 \bar{c} \cos \varphi
$$

$$
\bar{c}=c_{0}\left[\alpha\left(\bar{\gamma}^{\mathrm{p}}\right)^{\beta}+1\right]
$$

where, $H$ is hardening parameter; $\bar{c}$ is equivalent cohesive force. The yielding condition in this study considers influence of plastic strain hardening and damage degradation on internal cohesive effect of rocks. It is assumed that equivalent cohesive force is the power function of equivalent plastic deformation. $c_{0}$ is the cohesive force to be overcome by initial yielding; $\alpha$ and $\beta$ are plastic strain and hardening coefficients.

When destruction happens, $\bar{\gamma}^{p}=\bar{\gamma}_{c}^{p}$ namely, cohesive force $c$ meets the following formula:

$$
c=\bar{c}\left(1-\omega_{\mathrm{c}}\right)=c_{0}\left[\alpha\left(\bar{\gamma}_{\mathrm{c}}^{\mathrm{p}}\right)^{\beta}+1\right]\left(1-\omega_{\mathrm{c}}\right)
$$

Where, $\omega_{c}$ is the damage value at corresponding destruction point; $\bar{\gamma}_{c}^{p}$ is the equivalent plastic deformation in destruction and it corresponds to destruction strength. Destruction points are $\mathrm{E}$ and $\mathrm{D}$ on the curve in Figure 1.

$\omega_{c}$ can be calculated by formula (22):

$$
\omega_{\mathrm{c}}=1-\exp \left[-a\left(\bar{\gamma}_{\mathrm{c}}^{\mathrm{p}}\right)^{b}\right]
$$

According to formula (29), equivalent cohesive force $\bar{c}$ in formula (28) can be calculated:

$$
\bar{c}=\frac{c\left[\alpha\left(\bar{\gamma}^{\mathrm{p}}\right)^{\beta}+1\right]}{\left[\alpha\left(\bar{\gamma}_{\mathrm{c}}^{\mathrm{p}}\right)^{\beta}+1\right]\left(1-\omega_{\mathrm{c}}\right)}
$$

To avoid getting too large dilatancy deformation, nonrelevant yielding conditions are used. In other words, yielding function is not coincident with plastic potential function. While considering influence of damage, plastic potential function is:

$$
\begin{aligned}
g_{\mathrm{p}}\left(\sigma_{i j}^{\prime}\right) & =g_{\mathrm{p}}\left(\sigma_{i j}, \omega\right)=\sigma_{1}^{\prime}(1-\sin \psi)-\sigma_{3}^{\prime}(1+\sin \psi) \\
& =\left[\sigma_{1}(1-\sin \psi)-\sigma_{3}(1+\sin \psi)\right] /(1-\omega)
\end{aligned}
$$

where, $\psi$ is dilatancy angle; $g_{\mathrm{p}}$ is plastic potential function.

The damage compatibility condition of dilatancy angle $\psi$ and internal friction angle $\varphi$ is:

$\psi=(1-\omega) \varphi$

From formula (33), it is known that dilatancy angle gradually decreases following damage process. Its value contains influence of damage degradation. Namely, the value of dilatancy angle is not casual.

(3) Constitutive relation

(1) Common relational expressions

As for loading process:

$\mathrm{d} \sigma_{i j}^{\prime}=\mathrm{d}\left(\frac{\sigma_{i j}}{1-\omega}\right)=D_{i j k l}^{\mathrm{e}}\left(\mathrm{d} \varepsilon_{k l}-\mathrm{d} \varepsilon_{k l}^{p}\right)$

$\mathrm{d} \varepsilon_{i j}^{\mathrm{p}}=\lambda \frac{\partial g_{\mathrm{p}}\left(\sigma_{i j}^{\prime}\right)}{\partial \sigma_{i j}^{\prime}}$

$\lambda=\frac{1}{\eta} \frac{\partial f_{\mathrm{p}}}{\partial \sigma_{i j}^{\prime}} \mathrm{d} \sigma_{i j}^{\prime}$

where, $\eta$ is hardening modulus.

When $f_{p}$ in formula (26) meets consistency condition, $\mathrm{d} f_{p}=0$ namely, the following formula can be got:

$\eta=-\frac{\partial f_{\mathrm{p}}\left(\sigma_{i j}^{\prime}\right)}{\partial H} \frac{\partial H}{\partial \varepsilon_{i j}^{\mathrm{p}}} \frac{\partial g_{\mathrm{p}}\left(\sigma_{i j}^{\prime}\right)}{\partial \sigma_{i j}^{\prime}}$ 
While unloading, damage variable is invariable and meets the following formula:

$\mathrm{d} \sigma_{i j}=\left(1-\omega_{\mathrm{r}}\right) D_{i j k l}^{\mathrm{e}} \mathrm{d} \varepsilon_{k l}^{\mathrm{e}}=D_{i j k l}^{\omega} \mathrm{d} \varepsilon_{k l}^{\mathrm{e}}$

where, $D_{i j k l}^{\omega}$ is damage elastic rigidity tensor; $\omega_{r}$ is the largest damage variable in loading history before unloading.

Formulas (22)-(37) constitute the closed equation set of constitutive model of elastic-plastic statistic damage. Common constitutive relational expression is given.

(2) Constitutive relational expressions in conventional triaxial compression test

As for loading process:

The relation between axial stress and axial strain is:

$$
\begin{aligned}
& \mathrm{d}\left(\sigma_{1}-\sigma_{3}\right)=\mathrm{d} \sigma_{1}=E(1-\omega) \cdot \mathrm{d} \varepsilon_{1}^{\mathrm{e}}-\mathrm{d} \omega \cdot E \varepsilon_{1}^{\mathrm{e}} \\
& =\left[\mathrm{d} \varepsilon_{1}^{\mathrm{e}}-a b\left(\bar{\gamma}^{\mathrm{p}}\right)^{b-1} \cdot \varepsilon_{1}^{\mathrm{e}}\right] \cdot E \cdot \exp \left[-a\left(\bar{\gamma}^{\mathrm{p}}\right)^{b}\right] \\
& \mathrm{d} \varepsilon_{1}^{\mathrm{e}}=\mathrm{d} \varepsilon_{1}-\mathrm{d} \varepsilon_{1}^{\mathrm{p}}=\mathrm{d} \varepsilon_{1}-\lambda(1-\sin \psi) \\
& \lambda=\frac{(1-\sin \varphi)\left[\mathrm{d} \sigma_{1}(1-\omega)-\sigma_{1} \mathrm{~d} \omega\right]+(1+\sin \varphi) \sigma_{3} \omega}{\eta}
\end{aligned}
$$

$\eta=\frac{8 c_{0} \alpha \beta\left(\bar{\gamma}^{\mathrm{p}}\right)^{\beta-1} \cdot \cos \varphi}{3\left(\bar{\gamma}_{\mathrm{c}}^{\mathrm{p}}\right)^{\beta}}$

The relation between axial strain and volume strain:

$\mathrm{d} \varepsilon_{\mathrm{v}}^{\mathrm{e}}=(1-2 \mu) \mathrm{d} \varepsilon_{1}^{\mathrm{e}}$

$\mathrm{d} \varepsilon_{\mathrm{v}}^{\mathrm{p}}=\mathrm{d} \varepsilon_{1}^{\mathrm{p}}+2 \mathrm{~d} \varepsilon_{3}^{\mathrm{p}}=-\frac{1+3 \sin \psi}{1-\sin \psi} \cdot \mathrm{d} \varepsilon_{1}^{\mathrm{p}}$

$\mathrm{d} \varepsilon_{\mathrm{v}}=\mathrm{d} \varepsilon_{\mathrm{v}}^{\mathrm{e}}+\mathrm{d} \varepsilon_{\mathrm{v}}^{\mathrm{p}}=(1-2 \mu) \mathrm{d} \varepsilon_{1}^{\mathrm{e}}-\frac{1+3 \sin \psi}{1-\sin \psi} \cdot \mathrm{d} \varepsilon_{1}^{\mathrm{p}}$

While unloading, elasticity modulus meets the following formula:

$\mathrm{d}\left(\sigma_{1}-\sigma_{3}\right)=\left(1-\omega_{\mathrm{r}}\right) E \mathrm{~d} \varepsilon_{1}^{\mathrm{e}}=E_{\omega} \mathrm{d} \varepsilon_{1}^{\mathrm{e}}$

where, $\mu$ is a constant considering small variation of Poisson's ratio of rocks; elasticity modulus $E$ meets formula (14); $E_{\omega}$ is elasticity modulus of damage.

\subsection{Obtaining of parameters of constitutive model}

From formulae (22)-(45), it is known that independent parameters of constitutive model to be determined are damage parameters, yielding parameters and elastic parameters. Damage parameters are $n_{1}, n_{2}, n_{3}$ and $n_{4}$; yielding parameters are: cohesive force $c$ to be overcome while destroying rock, internal friction angle $\varphi$ of rock and strain hardening indexes $\alpha$ and $\beta$ of plastic flow; elastic parameters are: elasticity modulus $E$ (determined by $k_{1}$ and $k_{2}$ in formula (14)) and Poisson's ratio $\mu$. There are 11 independent parameters in the model obtained from conventional triaxial compression test.
Elasticity modulus $E$ and Poisson's ratio $\mu$ are determined by straight-line segment of test curve. They are calculated according to formula (39) and formula (40). $k_{1}$ and $k_{2}$ are determined by formula (14)

Generally, stress peak point corresponding to damage strength is used to determine cohesive force $c$ and internal friction angle $\varphi$ of rocks. The cohesive force $c_{0}$ that needs to be overcome by initial yielding, namely initial yielding point, corresponds to the elastic limit point $\mathrm{B}$ or $\mathrm{C}$ in Figure 1. Assuming difference of destruction limit stresses got from test curve is $\sigma_{1}-\sigma_{3}=Y_{\mathrm{c}}$, difference of elastic limit stresses is $\sigma_{1}-\sigma_{3}=Y_{0}$ and confining pressure is $\sigma_{2}=\sigma_{3}=S, Y_{\mathrm{c}}, Y_{0}$ and $S$ will meet the following formulae according to formula (26)

$$
\begin{aligned}
& \left(Y_{\mathrm{c}}+S\right)(1-\sin \varphi)-S(1+\sin \varphi)=2 c \cos \varphi \\
& \left(Y_{0}+S\right)(1-\sin \varphi)-S(1+\sin \varphi)=2 c_{0} \cos \varphi
\end{aligned}
$$

From formula (47), it is known that cohesive force $c$ and internal friction angle $\varphi$ at the time of destruction can be got by regression analysis of formula (47) after difference $Y_{c}$ of destruction limit stresses is got by test and when confining pressure $\mathrm{S}$ is a constant.

The following formula can be got from formulae (47) and (48):

$c_{0}=c-\frac{\left(Y_{\mathrm{c}}-Y_{0}\right)(1-\sin \varphi)}{2 \cos \varphi}$

After $c$ and internal friction angle $\varphi$ are determined, cohesive force $c_{0}$ at initial yielding can be calculated according to (49).

From formula (46), it is known that elastic modulus of damage is calculated below when unloading at destruction stress point:

$E_{\omega_{\mathrm{c}}}=E\left(1-\omega_{\mathrm{c}}\right)$

where, $E_{\omega_{c}}$ is elastic modulus of damage corresponding to destruction stress point. $(50)$ :

The following formula can be got from formulae (29) and

$\ln \left(\frac{E}{E_{\omega_{\mathrm{c}}}} \cdot \frac{c}{c_{0}}-1\right)=\ln \alpha+\beta \ln \bar{\gamma}_{\mathrm{c}}^{\mathrm{p}}$ $(50)$

The following formula can be got from formulae (46) and

$\ln \left[\ln \left(\frac{E}{E_{\omega}}\right)\right]=\ln a+b \ln \left(\bar{\gamma}^{\mathrm{p}} / \mathrm{e}^{S}\right)$

In the function of different confining pressures $S$, plastic strain and plastic flow strain hardening indexes $\beta$ and $\alpha$ can be got in regression analysis of formula (51) combining above $E$, $c_{0}$ and $c$ after getting $E_{\omega_{c}}$ and corresponding $\bar{\gamma}_{c}^{p}$.

Under specific confining pressure $S$, according to different damage elastic model $E_{\omega}$ and its corresponding equivalent plastic strain $\bar{\gamma}^{p}$ on stress-strain relation curve, Weibull 
distribution parameters $a$ and $b$ can be got from fitting of formula (52). The 4 parameters $n_{1}, n_{2}, n_{3}$ and $n_{4}$ can be got by fitting $a$ and $b$ under different confining pressures according to formulae (24) and (25).

According to formulae (47)-(52), all parameters in constitutive model can be got after processing test data.

\section{VERIFICATION OF CONSTITUTIVE MODEL}

To verify the rationality of constitutive model in this paper, the test data in Zhao et al., is processed to get relevant model parameters [19-21]. The triaxial compression constitutive relation in formulae (39)-(45) is put into FLAC3D limited differences calculus program by programming to simulate conventional triaxial compression test. By numerical simulation, axial stress-strain curve and volume strain-axial strain curve under different confining pressures can be got in Figure 3. The main calculating parameters are: $c=9.8 \mathrm{MPa}$; $\varphi=41^{\mathrm{o}} ; \mu=0.3 ; k_{1}=32403.5, k_{2}=0.2 ; n_{1}=0.07, n_{2}=11.7, n_{3}=0.02$, $n_{4}=8.5$. From Figure 3, it is known that there is little difference between the forecast result based on constitutive model in this study and test result.

According to Figure 2 and Figure 3, comparing with MC model, nonlinearity of stress-strain relation before and after peak value is considered in the improved constitutive model. Comparing with elastic damage constitutive model, rock dilatancy is considered in the improved constitutive model. In addition, the improved constitutive model considers that brittle destruction transforms to be elastic-plastic destruction following increase of confining pressure. In general, the improved constitutive model is more applicable, sufficiently considering rocks' deformation and destruction character.

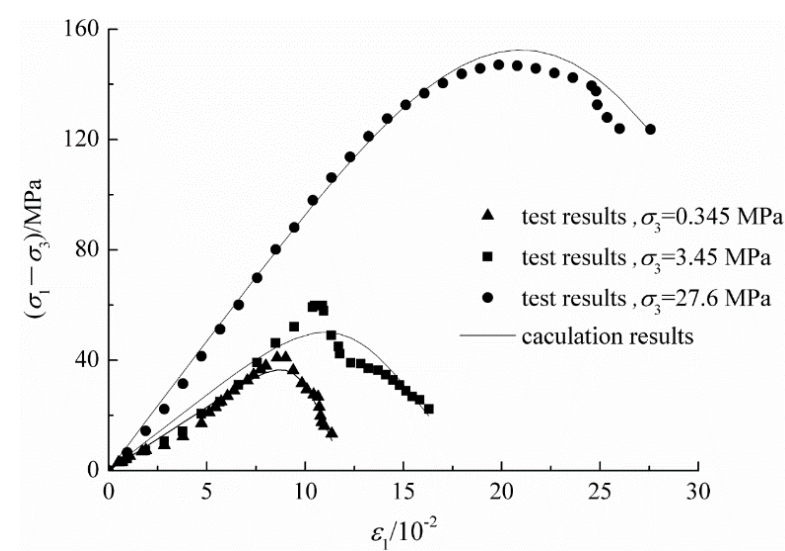

(a) Axial stress-strain relation

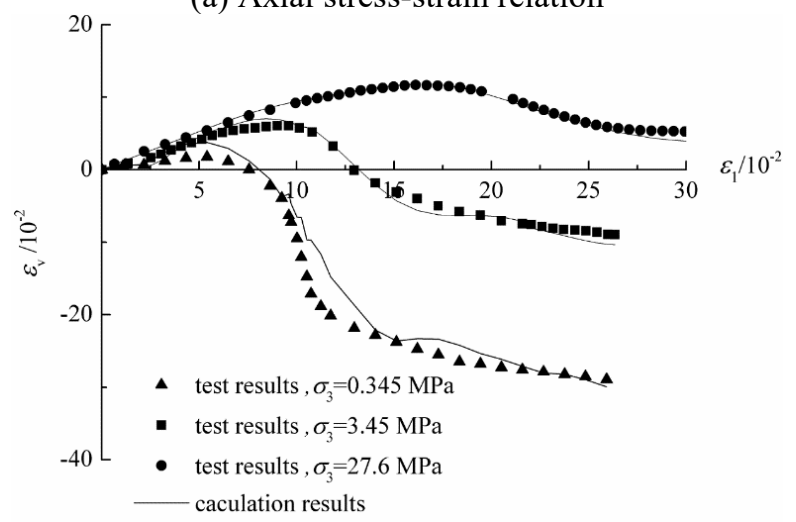

(b) Volume strain-axial strain relation

Figure 3. Result comparisons

\section{CONCLUSIONS}

The change law of rocks' stress-strain relations under conventional triaxial compression test conditions is summarized in this paper. It evaluates advantages and disadvantages of existing constitutive models, improves elastic damage constitutive model on the basis, puts forward constitutive model of elastic-plastic statistic damage, offers the method of getting model parameters and makes comparison between test result and numerical calculation result. The following main conclusions are obtained:

(1) Damage degradation and plastic flow exist in rock deformation and destruction process. Classical elastic-plastic constitutive model ignores mechanical damage degradation caused by internal defect of rocks. Elastic damage model ignores the irreversible deformation caused by plastic flow and exaggerates elastic deformation. So the former cannot reflect strain softening of rocks; the latter does not involve dilatancy and irreversible deformation. Therefore, influence of damage function and plastic flow shall be simultaneously considered while building constitutive model.

(2) It is assumed that plastic flow follows damage degradation and damage extension is restrained by confining pressure. At the same time, damage extension influences plastic deformation. The improved constitutive model considers the effect of coupling between damage function and plastic flow, which contributes to properly explaining the mechanism of rock deformation and destruction.

(3) The improved constitutive model can well and comprehensively reflect rocks' characters, including strain softening, dilatancy deformation and the transformation from brittle destruction to elastic-plastic destruction of rocks with the increase of confining pressure. Therefore, comparing with classical elastic damage model, the improved constitutive model can be more applicable. Besides, the number of calculating parameters in the improved model is small. There are 11 independent parameters. All parameters of the model can be obtained after making statistic analysis on triaxial test result. So the improved model can be used conveniently.

\section{ACKNOWLEDGEMENTS}

This research was financially supported in part by the National Natural Science Foundation of China (Grant No. 51274157 and No. 51378514)

\section{REFERENCES}

[1] Zhang M, Wang F, Yang Q. (2013). Statistical damage constitutive model for rocks based on triaxial compression tests. Chinese Journal of Geotechnical Engineering 35(11): 1965-1971.

[2] Chen JH, Zhang JS, Li XP. (2016). Model of rock blasting-induced damage considering integrity of rock mass and its application. Chinese Journal of Geotechnical Engineering 38(5): 857-866. https://doi.org/10.11779/CJGE201605011

[3] Chen L, Liu JF, Wang CP. (2013). Elastoplastic damage model of beishan deep granite. Chinese Journal of Rock Mechanics and Engineering 32(2): 289-298.

[4] Yuan XP, Liu HY, Wang ZQ. (2013). Crack-mechanics damage and plasticity for rock-like materials under 
compressive loading. Chinese Journal of Computational Mechanics 30(1): 149-155.

[5] Cao RL, He SH, Wei J. (2013). Study of modified statistical damage softening constitutive model for rock considering residual strength. Rock and Soil Mechanics 34(6): 1652-1660+1667.

[6] Yuan XP, Liu HY, Wang ZQ. (2012). Study of elastoplastic damage constitutive model of rocks based on Drucker-Prager criterion. Rock and Soil Mechanics 33(4): 1103-1108.

[7] Yuan XP, Liu HY, Wang ZQ. (2012). An interacting crack-mechanics based model for elastoplastic damage model of brittle materials under compression. Chinese Journal of Solid Mechanic 58(6): 92-102. https://doi.org/10.1016/j.ijrmms.2012.09.007

[8] Liu QJ, Yang LD, Cao WG. (2005). Statistical damage constitutive model for rock and back analysis of its papameters. Chinese Journal of Rock Mechanics and Engineering 24(4): 616-621. https://doi.org/10.3321/j.issn:1000-6915.2005.04.012

[9] Du Q, Wang C, Zhao GM. (2015). Development of constitutive model of fractured rock mass based on strain softening effect and its application. Journal of Yangtze River Scientific Research Institute 32(11): 82-86, 92.

[10] Yin YQ. (1995). On rock plasticity, damage and their constitutive formulation. Scientia Geologica Sinica 30(1): 63-70.

[11] Bale J, Valot E, Monin M, Polit O, Bathias C, Soemardi T. (2016). Experimental analysis of thermal and damage evolutions of DCFC under static and fatigue loading. Revue des Composites et des Materiaux Avances 26(2): $165-184$

[12] Yang SQ, Xu WY, Wei LD. (2004). Statistical constitutive model for rock damage under uniaxial compression and its experimental study. Journal of Hohai University (Natural Sciences) 32(2): 200-203.
[13] Wang JX, Jing AN. (2015). An elastoplastic damage constitutive model of rock and its application to tunnel engineering. Rock and Soil Mechanics 36(4): 11471158 .

[14] Wei LD, Xu WY, Yang CH. (2004). Study on elastoplastic constitutive model of rock with statistical damage. Chinese Journal of Rock Mechanics and Engineering 23(12): 1971-1975.

[15] Huang GM, Huang RQ. (1996). Coupling constitutive model between elastoplasticity and damage of rock. Journal of Xian Mining Institute 16(4): 40-45.

[16] Zhao JK, Zhang ZM, Liu ZQ. (2008). 3D numerical simulation of elasto-plastic damage and failure process of marble rock. Chinese Journal of Geotechnical Engineering 30(9): 1309-1315.

[17] Cao WG, Li X. (2008). A new discussion on damage softening statistical constitutive model for rocks and method for determining its parameters. Rock and Soil Mechanics 29(11): 2952-2956.

[18] Cao WG, Zhao MH, Liu CX. (2005). Study on rectified method of mohr-coulomb strength criterion for rock based on statistical damage theory. Chinese Journal of Rock Mechanics and Engineering 24(40): 2403-2408.

[19] Zhao XG, Cai M, Cai MF. (2010). A rock dilation angle model and its verification. Chinese Journal of Rock Mechanics and Engineering 29(5): 970-980.

[20] Wang FC, Yi SJ. (2018). Feng zenhancement of cantilever beams with fibre-reinforcement plastic. Chemical Engineering Transactions 64: 85-90. https://doi.org/10.3303/CET1864015

[21] Liu HZ, Shi YH. (2018). Integrated design for biomass compression molding machine based on cad technology. Chemical Engineering Transactions 65: 217-222. https://doi.org/10.3303/CET1865037 